\title{
Dietary patterns in relation to hepatic fibrosis among patients with nonalcoholic fatty liver disease
}

This article was published in the following Dove Medical Press journal: Diabetes, Metabolic Syndrome and Obesity:Targets and Therapy

\section{Davood Soleimani,' Golnaz Ranjbar, ${ }^{1,2}$ Reza Rezvani,, ${ }^{1,3}$ Ladan Goshayeshi, ${ }^{4}$ Farkhonde Razmpour,' Mohsen Nematy' \\ 'Department of Nutrition, Faculty of Medicine, Mashhad University of Medical Sciences, Mashhad, Iran; ${ }^{2}$ Department of Biological and Environmental Sciences, Faculty of Life and Medical Sciences, University of Hertfordshire, Hatfield, UK; ${ }^{3}$ School of Nutrition, Faculté des Sciences de l'agriculture et de l'alimentation, Université Laval, Quebec, QC, Canada; ${ }^{4}$ Department of Gastroenterology and Hepatology, Faculty of Medicine, Mashhad University of Medical Sciences, Mashhad, Iran}

\section{Video abstract}

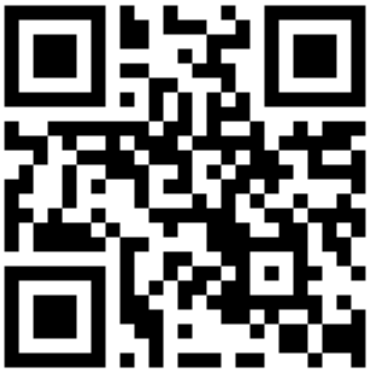

Point your SmartPhone at the code above. If you have a QR code reader the video abstract will appear. Or use: http://youtu.be/Z8pKXquiAp0

Correspondence: Mohsen Nematy Department of Nutrition, Mashhad Medical School, Paradise Daneshgah, Azadi Square, Mashhad, 91779-48564, Iran

Tel +9838002103

Fax +983800 2421

Email NematyM@mums.ac.ir
Background: Nonalcoholic fatty liver disease (NAFLD) is a multifactorial disorder that can progress to fibrosis. Several dietary patterns have been associated with histological features of NAFLD. However, little is known about the association between dietary patterns and hepatic fibrosis.

Purpose: The current study aimed at identifying the relationship between major dietary patterns and hepatic fibrosis among patients with NAFLD.

Subjects and methods: This cross-sectional study included 170 eligible subjects with NAFLD. Diet was evaluated using three 3-day dietary records during a 1-month period. Hepatic fibrosis was diagnosed using Fibroscan. Western, Iranian, and healthy dietary patterns were extracted using factor analysis.

Results: After adjustment for other risk factors, adherence to a Western dietary pattern was associated with the higher odds of fibrosis (OR: 4.21; 95\% CI: 1.63-8.31), whereas adherence to a healthy dietary pattern was associated with the lower odds of fibrosis (OR: $0.26 ; 95 \% \mathrm{CI}$ : 0.10-0.49). Among main food groups, high intake of red meat, hydrogenated fats, and soft drinks was associated with the higher risk of fibrosis, whereas tea and coffee consumption had a protective role in hepatic fibrosis independent of other risk factors.

Conclusion: The adherence to a healthy dietary pattern characterized by high intake of low-fat dairies, white meat, nuts, vegetables, fruits, and vegetable oils combined with coffee and tea consumption might be helpful in the nutritional strategies against hepatic fibrosis.

Keywords: liver diseases, NAFLD, fibrosis, factor analysis, diet, dietary pattern

\section{Introduction}

Nonalcoholic fatty liver disease (NAFLD) is known as the hepatic manifestation of metabolic syndrome and insulin resistance. ${ }^{1}$ NAFLD currently affects about $25.24 \%$ of adults and $7.6 \%$ of children in the general population, whereas obese adults and children contribute to $51.34 \%$ and $34.2 \%$ of worldwide predominance, respectively. ${ }^{2,3}$ NAFLD includes a wide spectrum of hepatocellular damage in the absence of excessive alcohol intake, from simple steatosis (nonalcoholic fatty liver [NAFL]) to steatohepatitis (nonalcoholic steatohepatitis [NASH]) that can progress to fibrosis in $64.2 \%$ of cases. ${ }^{4,5}$ The progression rate of fibrosis is predicted to be 0.07 and 0.14 stage/year in NAFL and NASH patients, respectively. ${ }^{5}$ To date, several therapeutic agents, such as pioglitazone and vitamin $\mathrm{E}$, have been proposed for the treatment and prevention of hepatic steatosis and steatohepatitis in NAFLD, but their efficacy and safety are still controversial.,

It is well established that NAFLD is a multifactorial disorder which could be driven by the interaction between genome and environmental factors. Diet as the 
main modifiable environmental factor has a causative role in NAFLD occurrence. ${ }^{8}$ A growing body of research has examined the relationship between the disease and individual dietary components. However, this approach discards many aspects of diet such as the interaction and cumulative effects of nutrients or foods ingested together. ${ }^{9}$ Currently, "dietary pattern analysis" is determined as a complementary approach to investigate overall dietary impact and overcome these limitations. Furthermore, dietary patterns are suggested to be easier for the public to translate into a realistic dietary intake and nutritional strategies. ${ }^{10}$ Thus, identifying the relationship between dietary patterns and features of NAFLD could be valuable to address the issue in these patients.

Recent studies reported that improving adherence to healthy dietary patterns such as "Mediterranean" and "Dietary Approaches to Stop Hypertension" diet, which are highlighted on whole grains, low-fat dairy products, vegetables, and fruits, may have inverse association with hepatic steatosis and steatohepatitis in NAFLD patients. ${ }^{11,12}$ Also, another study showed that the Mediterranean diet intervention could improve the hepatic steatosis. ${ }^{13}$ Conversely, unhealthy dietary patterns, such as Western food style, which emphasizes on the high intake of red meat, soft drinks, refined grains, high-fat dairy products, and processed meat, were positively associated with NAFLD incidence. ${ }^{14}$

Regardless of current extensive clinical and epidemiological studies, however, the evidence on the relation between dietary patterns and the risk of hepatic fibrosis among NAFLD is limited. Considering the high risk of fibrosis progression and the important role of diet in NAFLD pathogenesis or even treatment, the aim of the present study was to identify the relationship between major dietary patterns and risk of hepatic fibrosis among patients with NAFLD.

\section{Subjects and methods Subjects}

This cross-sectional study was carried out among patients with NAFLD in Mashhad, Iran. Participants were recruited from consecutive outpatients who attended the gastrointestinal clinic at Imam Reza hospital from August to October 2018. One hundred and seventy eligible patients were needed to be included in the final analysis according to previous recommendations for factor analysis in which ten subjects were needed for each food group. ${ }^{15}$ The inclusion criteria for participation in the study were as follows: 1) adult patients aged between 20 and 60 years; 2) the recent diagnosis of hepatic steatosis (in the last 6 months) with imagining techniques; 3) no significant alcohol consumption; 4) no other secondary causes of hepatic steatosis (eg, hepatitis $\mathrm{B}$ and $\mathrm{C}$ virus, autoimmune hepatitis, hemochromatosis, Wilson's disease, or hepatotoxic and corticosteroid medications); and 5) no change in eating behaviors since NAFLD diagnosis. The current study was conducted in accordance with the Declaration of Helsinki, and all patients completed the informed consent form. The study protocol was also approved by the Ethics Committee of Mashhad University of Medical Sciences, Mashhad, Iran (Number: IR.MUMS. MEDICAL.REC.1397.121).

\section{Dietary assessment}

Dietary information was obtained by three 3-day dietary records (3-DRs) during a 1-month period. All eligible patients were instructed to complete 3-DRs during consecutive days. A dietician calculated the amount of food items using Iranian household measures. Then, each food item was converted to a value of energy and other nutrients using Nutritionist IV software (N-Squared Computing, Salem, OR, USA). Dietary intake data were excluded in the final analysis if the energy intake was not within the normal range ( $800-4,200 \mathrm{kcal} /$ day). To obtain dietary patterns, food items were assigned into main food groups according to their nutritional similarities or culinary usage. We also considered some food items (tea, potato, and egg) as separated food groups because of specific nutrition profiles. Seventeen food groups were included in factor analysis (Table S1).

\section{Transient elastography}

Hepatic fibrosis was evaluated using Fibroscan machine (Echosens, Paris, France), as a noninvasive technique with high intra-/inter-observer agreement. ${ }^{16}$ Liver stiffness measurements (LSMs) were taken with the use of M probe of Fibroscan at fasting state with the supine position on the right lobe of the liver. All measurements were performed by one expert radiologist with one Fibroscan device at the same center. Each patient underwent one set of transient elastography examination. Only those examinations were considered reliable which had at least ten valid LSMs with their interquartile range/median ratio less than $30 \% .{ }^{16}$ The median value of LSMs more than $5.3 \mathrm{kPa}$ was considered as the optimal cut-off point (area under the receiver operating characteristic curve $=0.879$ ) for predicting hepatic fibrosis according to Meta-analysis of Histological Data in Viral Hepatitis classification. ${ }^{17}$ Also, Fibroscan machine with the implement of controlled attenuation parameter (CAP) is able to quantify the hepatic steatosis. Accord- 
ing to CAP cut-off points, hepatic steatosis was classified into four stages; CAP $<238 \mathrm{~dB} / \mathrm{m}$ for no steatosis, $238 \leq$ $\mathrm{CAP}<259 \mathrm{~dB} / \mathrm{m}$ for mild steatosis, $259 \leq \mathrm{CAP}<292 \mathrm{~dB} / \mathrm{m}$ for moderate steatosis, and CAP $\geq 292 \mathrm{~dB} / \mathrm{m}$ for severe steatosis. $^{18}$

\section{Biochemical assessment}

Blood specimens were taken from all patients after a 10-hour overnight fast. Serum samples were obtained from the coagulated blood after centrifugation and were immediately stored at $-20^{\circ} \mathrm{C}$ until their analysis. Serum alanine aminotransferase (ALT), aspartate aminotransferase (AST), gamma-glutamyl transferase (GGT), total cholesterol, triglycerides (TG), and fasting blood sugar were assayed photometrically with the use of commercial kits (Pars Azmoon, Tehran, Iran). We also measured the serum insulin level by the commercial kit (Commercial ELISA kit; Monobind, Lake Forest, CA, USA) and calculated insulin resistance using the homeostasis model assessment for insulin resistance according to the formula: (fasting glucose $[\mathrm{mmol} / \mathrm{L}] \times$ insulin $[\mathrm{m} \mathrm{IU} / \mathrm{L}]$ )/ 22.5.

\section{Other risk factors assessment}

Anthropometric assessment was administered by an expert nutritionist. Weight and height were measured to calculate body mass index (BMI). Body weight was measured on a digital scale (Seca 704, Germany) to the nearest $0.1 \mathrm{~kg}$ with the subjects in light clothing and without shoes. Height was measured to the nearest $0.1 \mathrm{~cm}$ by means of a stadiometer attached to the wall. Then, BMI was calculated as weight (in kilograms) divided by the square of the height (in meters). The body fat mass was measured at the standard condition with Bioelectrical Impedance Analysis system (Tanita BC418; Tanita Corporation, Tokyo, Japan). Furthermore, waist circumference (WC) was measured with a flexible tape to the nearest $0.1 \mathrm{~cm}$ at the minimum circumference between the iliac crest and the rib cage. A self-administered questionnaire was used to assess socio-demographic characteristics and medical history. The intensity of physical activity (metabolic equivalent) was calculated by the use of the International Physical Activity Questionnaire according to its guideline.

\section{Statistical analysis}

Data were analyzed with SPSS software version 16.0 (SPSS, Inc., Chicago, IL, USA). The normal distribution of continues variables was assessed with the Kolmogorov-Smirnov test. The independent-sample $t$-test (for normally distributed variables), Mann-Whitney U test (for abnormally distributed variables), and chi-squared test (for categorical variables) were performed to compare the general characteristics according to hepatic fibrosis status. Multivariable-adjusted OR obtained from the logistic regression model was used to determine the association between food groups and hepatic fibrosis.

Factor analysis was applied to extract dietary patterns from 17 food groups. The intercorrelation among food groups was determined with the Kaiser-Meyer-Olkin and Bartlett's sphericity test. The number of retained dietary patterns was decided by the eigenvalue more than 1.5 and shape of scree plot based on a previous study. ${ }^{19}$ These retained dietary patterns were rotated with Varimax rotation test in order to make an easier interpretation. The scores of each dietary pattern were computed by summing up intakes of all food groups weighted by their factor loadings. The components of each dietary pattern were determined according to the rotated factor loading (absolute) more than 0.3. We also categorized patients to low, medium, and high adherence to each dietary pattern based on tertiles of dietary pattern scores.

Age-, gender-, and energy-adjusted mean of nutrient intakes across tertiles of each dietary pattern was compared using the analysis of covariance. Furthermore, Bonferroni's post hoc test was used to make a comparison between the first and last tertile. The association of dietary patterns with hepatic fibrosis status was determined using the binary logistic regression. We defined three models to compute multivariable-adjusted ORs. The first model was adjusted for age, gender, BMI, WC, smoking (yes, no), diabetes, education (categorical), and anti-diabetic medication (yes, no) and dietary supplement use (yes, no). The second model was further adjusted for hepatic steatosis status (categorical). Finally, the third model was additionally adjusted for the physical activity (metabolic equivalent hours/week) and energy intake.

\section{Results}

Two hundred and twenty-two (74\%) of the 300 patients enrolled in this study met all the inclusion criteria, of whom 189 eligible participants returned the completed 3-DRs (response rate: $85.1 \%$ ) and underwent assessment phase. Among transient elastography examinations, $3.2 \%$ of them had poorly reliable results. A total of 170 eligible participants with reliable data for all measurements were included in factor analysis (Figure 1).

General characteristics of NAFLD patients according to hepatic fibrosis status are presented in Table 1 . The rate of diabetes and severe steatosis was significantly higher in the patients with fibrosis than those without fibrosis. The patients 


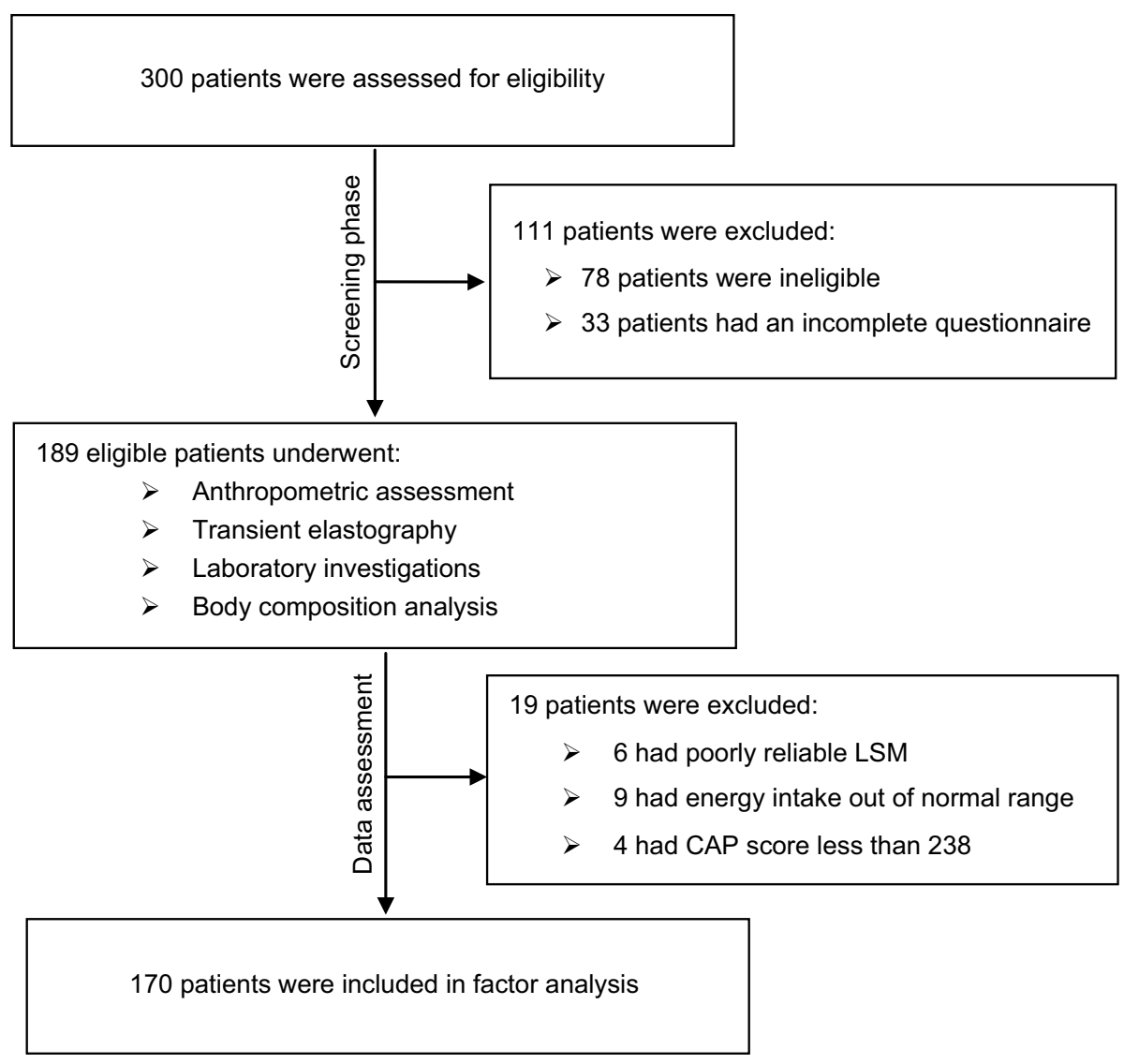

Figure I The study flowchart.

Abbreviations: CAP, controlled attenuation parameter; LSM, liver stiffness measurement.

with hepatic fibrosis showed a significantly lower educational level than those without fibrosis. In patients with fibrosis, a significantly higher mean of weight, BMI, AST, ALT, GGT, TG, WC, and body fat mass was observed as compared to patients without fibrosis $(P<0.05)$. No statistically significant difference was observed in other parameters between both groups.

The factor loading matrix of dietary patterns is presented in Table 2. Three major dietary patterns were identified using factor analysis with a Kaiser-Mayer-Olkin criterion 0.600 and Bartlett's sphericity test $<0001$. The first dietary pattern explaining $14.89 \%$ of total variance was labeled as Western, comprising high intake of red and white meats, refined grains, potato, eggs, and soft drinks. The second dietary pattern explained $9.57 \%$ of total variance and was labeled as Iranian, comprising high intake of high-fat dairies, refined grains, white meat, legumes, tea and coffee, sugars, and hydrogenated fats. The third dietary pattern explaining $8.51 \%$ of total variance was labeled as healthy, comprising high intake of low-fat dairies, white meat, vegetables, fruits, nuts, and vegetable oils.
Multivariable-adjusted risk of hepatic fibrosis across tertiles of food groups is illustrated in Figure 2. A significantly higher risk of fibrosis was observed in the highest tertile of red meat (OR: 2.19; 95\% CI: 1.21-3.74), hydrogenated fats (OR: 2.58; 95\% CI: 1.48-4.98), and soft drinks (OR: 2.21; 95\% CI: 1.15-4.23), as compared to the lowest tertile. In contrast, the patients in the highest tertile for coffee and tea intake showed a significantly lower risk of fibrosis (OR: 0.38; 95\% CI: 0.17-0.71) than those in the lowest tertile. The other food groups showed no significant association with hepatic fibrosis. Further details are provided in Table S2.

Multivariable-adjusted means for dietary intakes across tertiles of dietary pattern scores are displayed in Table 3. In the Western dietary pattern, participants with higher adherence had higher intakes of energy, total fat, saturated fatty acids (SFAs), and monounsaturated fatty acids (MUFAs), but lower intakes of protein, carbohydrate, vitamin C, and dietary fiber. However, we observed no significant difference in the unsaturated to saturated fatty acids ratio across the tertiles of this pattern. Higher adherence to Iranian dietary pattern was associated with higher intakes of energy, carbohydrate, 
Table I General characteristics of NAFLD patients with and without fibrosis

\begin{tabular}{|c|c|c|c|}
\hline Variables & Nonfibrosis $(n=76)$ & Fibrosis $(n=94)$ & $P$-value \\
\hline Age, years & $39.06 \pm 13.27$ & $39.69 \pm|2.8|$ & $0.76^{a}$ \\
\hline Male, $n \%$ & 30.6 & 41.5 & $0.17^{\mathrm{b}}$ \\
\hline Weight, kg & $89.7 \pm 14.9$ & $98 \pm 20.5$ & $0.0 \mathrm{I}^{\mathrm{a}}$ \\
\hline $\mathrm{BMI}, \mathrm{kg} / \mathrm{m}^{2}$ & $31.3 \pm 4.5$ & $34 \pm 5.7$ & $0.003^{a}$ \\
\hline CVD, n \% & 11.3 & 21.3 & $0.11^{\mathrm{b}}$ \\
\hline Diabetes mellitus, $n$ \% & 6.5 & 22.3 & $0.008^{b}$ \\
\hline Smoker, $\mathrm{n} \%$ & 17.7 & 20.2 & $0.72^{\mathrm{b}}$ \\
\hline University education, $\mathrm{n} \%$ & 20.2 & 8.1 & $0.04^{\mathrm{b}}$ \\
\hline Current anti-diabetic drugs use, $n \%$ & 19.7 & 28.7 & $0.17^{\mathrm{b}}$ \\
\hline Current dietary supplement use, $n \%$ & $\mathrm{II} .8$ & 13.8 & $0.70^{\mathrm{b}}$ \\
\hline Body fat mass, kg & $29.30 \pm 5.94$ & $34.1 \pm 7.3$ & $0.04^{\mathrm{c}}$ \\
\hline Waist circumference, $\mathrm{cm}$ & $107 \pm 16.67$ & $114 \pm 9.8$ & $0.02^{c}$ \\
\hline Severe hepatic steatosis, $\mathrm{n} \%$ & 16.1 & 33 & $0.00 \mathrm{I}^{\mathrm{b}}$ \\
\hline Physical activity, MET-h/week & $9.67 \pm 5.7$ & $10.31 \pm 4.9$ & $0.7 I^{c}$ \\
\hline Aspartate aminotransferase, IU.dL- ${ }^{\prime}$ & $40.25 \pm 7.43$ & $47.06 \pm 11.9$ & $0.00 \mathrm{I}^{\mathrm{a}}$ \\
\hline Alanine aminotransferase, IU·dL-I & $39.80 \pm 8.56$ & $53.88 \pm 19.52$ & $0.00 \mathrm{I}^{\mathrm{c}}$ \\
\hline Gamma-glutamyl transferase, IU·dL-' & $43.64 \pm 7.04$ & $50.78 \pm 13.57$ & $0.005^{\mathrm{a}}$ \\
\hline Triglyceride, $\mathrm{mg} \cdot \mathrm{dL}-{ }^{\prime}$ & $161 \pm 56.61$ & $188.44 \pm 66.12$ & $0.004^{c}$ \\
\hline Total cholesterol, $\mathrm{mg} \cdot \mathrm{dL}-{ }^{\prime}$ & $224.42 \pm 40.42$ & $227.02 \pm 38.84$ & $0.727^{a}$ \\
\hline Fasting blood sugar, $\mathrm{mg} \cdot \mathrm{dL}-{ }^{\prime}$ & $98.20 \pm 19.87$ & $103.32 \pm 26.5$ & $0.256^{\mathrm{a}}$ \\
\hline HOMA-IR & $3.37 \pm 2.5 \mathrm{I}$ & $4.64 \pm 2.86$ & $0.056^{c}$ \\
\hline
\end{tabular}

Notes: ${ }^{P}$-values were calculated using independent-sample $t$-test. ${ }^{b} P$-values were calculated using chi-squared test. ${ }^{C P}$-values were calculated using Mann-Whitney $U$ test. Data are reported as mean $\pm S D$ or percentage as appropriate.

Abbreviations: BMI, body mass index; CVD, cardiovascular disease; HOMA-IR, homeostasis model assessment for insulin resistance; MET-h, metabolic equivalent hours; NAFLD, nonalcoholic fatty liver disease.

Table 2 Factor loading matrix for major dietary patterns identified in NAFLD patients

\begin{tabular}{|l|l|l|l|}
\hline Food groups & $\begin{array}{l}\text { Western } \\
\text { pattern }\end{array}$ & $\begin{array}{l}\text { Iranian } \\
\text { pattern }\end{array}$ & $\begin{array}{l}\text { Healthy } \\
\text { pattern }\end{array}$ \\
\hline High-fat dairies & & $\mathbf{0 . 7 1 7}$ & \\
\hline Low-fat dairies & & & $\mathbf{0 . 6 6 2}$ \\
\hline Red meat & $\mathbf{0 . 3 0 5}$ & & \\
\hline White meat & $\mathbf{0 . 4 4 5}$ & $\mathbf{0 . 4 4 0}$ & $\mathbf{0 . 4 9 0}$ \\
\hline Refined grains & $\mathbf{0 . 7 4 2}$ & $\mathbf{0 . 4 3 8}$ & \\
\hline Whole grains & & -0.226 & \\
\hline Potato & $\mathbf{0 . 5 9}$ & 0.203 & \\
\hline Eggs & $\mathbf{0 . 5 9 0}$ & & \\
\hline Legumes & 0.223 & $\mathbf{0 . 3 2 3}$ & \\
\hline Nuts & & & $\mathbf{0 . 3 1 8}$ \\
\hline Vegetables & & & $\mathbf{0 . 4 3 2}$ \\
\hline Fruits & & & $\mathbf{0 . 4 4 9}$ \\
\hline Coffee and tea & & $\mathbf{0 . 5 9 3}$ & \\
\hline Sugars & 0.238 & $\mathbf{0 . 5 4 2}$ & \\
\hline Soft drinks & $\mathbf{0 . 6 6 9}$ & & \\
\hline Vegetable oils & & & $\mathbf{0 . 4 6 0}$ \\
\hline Hydrogenated fats & & $\mathbf{0 . 5 9 2}$ & \\
\hline Variance explained & $14.89 \%$ & $9.57 \%$ & $8.51 \%$ \\
\hline Notes Absoly val & &
\end{tabular}

Notes: Absolute values less than 0.2 are not displayed for simplicity. Numbers in bold indicated main food groups with significant correlation $(>0.3)$ within each dietary pattern.

Abbreviation: NAFLD, nonalcoholic fatty liver disease. and protein. A significant downward trend was shown in the intakes of total fat, MUFAs, and SFAs with increasing adherence to this pattern. The ratio of unsaturated to saturated fatty acids showed no significant differences across the tertiles of this pattern. In contrast with other patterns, there was no increase in energy intake with increasing adherence to healthy dietary pattern. However, subjects in the high tertile of healthy dietary pattern consumed more amounts of protein, fiber, vitamin $\mathrm{E}$, and vitamin $\mathrm{C}$, but less carbohydrate, as compared to subjects in the low tertile of this pattern.

The risk of hepatic fibrosis across tertiles of the dietary pattern scores is presented in Table 4. The adherence to Western dietary pattern did not show statistically significant association with hepatic fibrosis. But, this association became statistically significant after adjustment for confounding factors. In the first model, high adherence to the Western pattern was associated with higher odds of fibrosis (OR: 2.38; 95\% CI: 1.12-5.63) than low adherence. In the second model, following further adjustment for hepatic steatosis status, a significant upward trend in the odds of fibrosis was seen with increasing the adherence to this pattern $(P$-trend $<0.05)$. Conversely, the adherence to the 


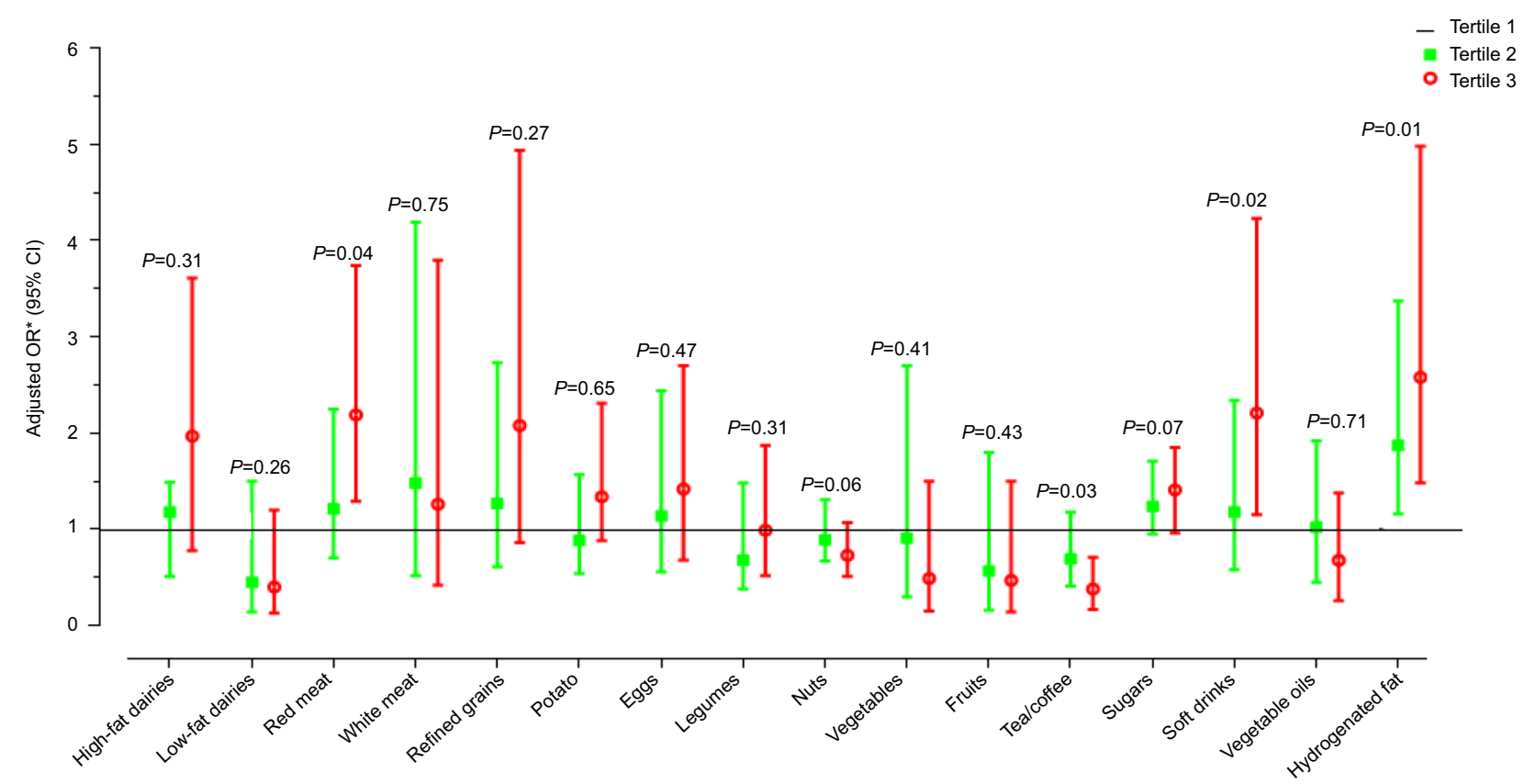

Figure 2 Multivariable-adjusted odds ratio for hepatic fibrosis across tertiles of main food groups.

Notes: *Adjusted for sex, BMI, WC, smoking, education, diabetes, physical activity, hepatic steatosis status, energy intake, and anti-diabetic drugs and dietary supplement use. Abbreviations: BMI, body mass index; WC, waist circumference.

Table 3 Multivariable-adjusted means ${ }^{\text {a }}$ for dietary intakes across tertiles $(T)$ of dietary pattern scores

\begin{tabular}{|c|c|c|c|c|c|c|c|c|c|}
\hline \multirow[b]{2}{*}{ Dietary intakes } & \multicolumn{2}{|c|}{ Western pattern } & \multirow[b]{2}{*}{$P$-trend } & \multicolumn{2}{|c|}{ Iranian pattern } & \multirow[b]{2}{*}{$P$-trend } & \multicolumn{2}{|c|}{ Healthy pattern } & \multirow[b]{2}{*}{$P$-trend } \\
\hline & T I (lowest) & T3 (highest) & & TI (lowest) & T3 (highest) & & TI (lowest) & T3 (highest) & \\
\hline Energy $\left(\mathrm{kcal} \cdot \mathrm{d}^{-1}\right)$ & $1669 \pm 106^{b}$ & $2258 \pm 99$ & 0.001 & $1514 \pm 102^{b}$ & $2404 \pm 96$ & 0.001 & $1886 \pm 109$ & $2046 \pm 103$ & 0.294 \\
\hline Carbohydrate $\left(\mathrm{g} \cdot \mathrm{d}^{-1}\right)$ & $285 \pm 6.7$ & $249 \pm 6.4$ & 0.001 & $252 \pm 7.3$ & $279 \pm 7.1$ & 0.016 & $275 \pm 6.3^{b}$ & $243.6 \pm 5.9$ & 0.001 \\
\hline Fat $\left(g \cdot d^{-1}\right)$ & $5 \mathrm{I} \pm 2.8^{\mathrm{b}}$ & $71.2 \pm 2.7$ & 0.001 & $69.7 \pm 3.1^{b}$ & $53.2 \pm 3.1$ & 0.001 & $61.5 \pm 2.8^{\mathrm{b}}$ & $68.4 \pm 2.7$ & 0.081 \\
\hline Protein $\left(g \cdot d^{-1}\right)$ & $72.6 \pm 2^{\mathrm{b}}$ & $62.8 \pm 1.9$ & 0.001 & $63.5 \pm 2.1^{b}$ & $73.8 \pm 2$ & 0.001 & $59.5 \pm 1.78^{b}$ & $75.1 \pm 1.69$ & 0.001 \\
\hline MUFAs $\left(g \cdot d^{-1}\right)$ & $16.4 \pm 1.7^{b}$ & $24.3 \pm 1.1$ & 0.001 & $23.8 \pm 1.3^{b}$ & $17.5 \pm 1.2$ & 0.001 & $20.7 \pm 1.2$ & $23.2 \pm 1.1$ & 0.126 \\
\hline PUFAs $\left(\mathrm{g} \cdot \mathrm{d}^{-1}\right)$ & $10.2 \pm 1.18$ & $9.7 \pm 1.13$ & 0.78 & $10.5 \pm 1.2$ & $9.9 \pm 1.22$ & 0.754 & $10.2 \pm 1.09$ & $12.09 \pm 1.04$ & 0.22 \\
\hline SFAs $\left(g \cdot d^{-1}\right)$ & $19.5 \pm 1.2^{b}$ & $30.9 \pm 1.1$ & 0.001 & $29.7 \pm 1.4^{b}$ & $20.6 \pm 1.3$ & 0.001 & $24.7 \pm 1.34$ & $27.15 \pm 1.28$ & 0.195 \\
\hline USFAs/SFAs & $1.35 \pm 0.08$ & $1.19 \pm 0.08$ & 0.189 & $1.24 \pm 0.08$ & $1.36 \pm 0.08$ & 0.11 & $1.33 \pm 0.07$ & $1.38 \pm 0.07$ & 0.63 \\
\hline Fiber $\left(g \cdot d^{-1}\right)$ & $27 \pm 1.33^{b}$ & $22.1 \pm 1.34$ & 0.015 & $22.4 \pm 1.47$ & $24.1 \pm 1.57$ & 0.49 & $20.9 \pm 1.3 \mathrm{I}^{\mathrm{b}}$ & $25.5 \pm 1.33$ & 0.017 \\
\hline Vitamin $E\left(m g \cdot d^{-1}\right)$ & $6.40 \pm 0.79$ & $6.42 \pm 0.85$ & 0.98 & $7.02 \pm 0.74$ & $5.21 \pm 0.74$ & 0.097 & $5.53 \pm 0.715^{b}$ & $7.7 I \pm 0.7$ & 0.034 \\
\hline Vitamin $C\left(\mathrm{mg} \cdot \mathrm{d}^{-1}\right)$ & $119.8 \pm 11.7^{b}$ & $81.4 \pm 12.5$ & 0.047 & $100 \pm 11.2$ & $108.2 \pm 11.1$ & 0.62 & $89.2 \pm 10.8^{b}$ & $126 \pm 10.6$ & 0.018 \\
\hline
\end{tabular}

Notes: aAdjusted for age, gender, and energy, obtained from analysis of covariance (ANCOVA). ${ }^{b}$ Significant difference between the first and last tertile, obtained from Bonferroni's post hoc test. Data are expressed as mean \pm standard error.

Abbreviations: MUFAs, monounsaturated fatty acids; PUFAs, polyunsaturated fatty acids; SFAs, saturated fatty acids; USFAs, unsaturated fatty acids.

healthy pattern was associated with a decreased risk of hepatic fibrosis. The subjects with high adherence to this pattern had lower odds of hepatic fibrosis (OR: 0.43; 95\% CI: 0.19-0.97) compared to those with low adherence. The strength of this negative association increased with further adjustment for confounding factors, such as hepatic steatosis. There was no significant association between Iranian dietary pattern and hepatic fibrosis not only in crude model but also in adjusted models.

\section{Discussion}

Three major dietary patterns were extracted in the current study, including Western, Iranian, and healthy. The adherence to the healthy pattern was inversely associated with hepatic fibrosis, whereas the Western pattern showed a positive association. Furthermore, intake of tea and coffee showed a protective role in hepatic fibrosis. To the best of our knowledge, the current study is the first to investigate 
Table 4 Odds ratios $(95 \% \mathrm{Cl})^{\text {a }}$ for hepatic fibrosis across tertiles $(\mathrm{T})$ of dietary pattern scores

\begin{tabular}{|c|c|c|c|c|}
\hline Dietary pattern & TI (low adherence) & T2 & T3 (high adherence) & P-trend \\
\hline \multicolumn{5}{|l|}{ Western pattern } \\
\hline Crude & Ref & I.4I (0.65-3.09) & $2.22(0.99-4.96)$ & 0.051 \\
\hline Model I & Ref & $1.35(0.64-2.63)$ & $2.38(1.12-5.63)$ & 0.073 \\
\hline Model 2 & Ref & $1.63(0.74-3.44)$ & $2.73(1.31-6.84)$ & 0.031 \\
\hline Model 3 & Ref & $2.04(\mathrm{I} .03-3.8 \mathrm{I})$ & $4.2 \mathrm{I}(\mathrm{I} .63-8.3 \mathrm{I})$ & 0.042 \\
\hline \multicolumn{5}{|l|}{ Iranian pattern } \\
\hline Crude & Ref & $\mathrm{I} .12(0.5 \mathrm{I}-2.5 \mathrm{I})$ & $0.66(0.30-1.45)$ & 0.298 \\
\hline Model I & Ref & $1.15(0.57-2.73)$ & $0.75(0.43-1.28)$ & 0.348 \\
\hline Model 2 & Ref & I.5I $(0.73-3.37)$ & $0.84(0.4 I-I .5 I)$ & 0.531 \\
\hline Model 3 & Ref & $\mathrm{I} .47(0.43-2.9 \mathrm{I})$ & $0.81(0.34-1.63)$ & $0.4 \mathrm{II}$ \\
\hline \multicolumn{5}{|l|}{ Healthy pattern } \\
\hline Crude & Ref & $0.61(0.27-1.39)$ & $0.43(0.19-0.97)$ & 0.042 \\
\hline Model I & Ref & $0.51(0.23-0.98)$ & $0.37(0.19-0.73)$ & 0.023 \\
\hline Model 2 & Ref & $0.36(0.18-0.68)$ & $0.29(0.13-0.53)$ & 0.018 \\
\hline Model 3 & Ref & $0.37(0.15-0.72)$ & $0.26(0.10-0.49)$ & 0.011 \\
\hline
\end{tabular}

Notes: ${ }^{a}$ Odds ratios $(95 \% \mathrm{Cl})$ were obtained using binary logistic regression. Model I: adjusted for sex, age, BMI, WC, smoking, education, diabetes, and anti-diabetic medication and dietary supplement use. Model 2: model I plus the further adjustment for hepatic steatosis status. Model 3: model 2 plus additional adjustment for energy intake and physical activity.

Abbreviations: BMI, body mass index; WC, waist circumference.

the association between major dietary patterns and hepatic fibrosis in NAFLD.

Our findings showed a significant association between hepatic fibrosis and severity of steatosis. It is well established that this relationship might be mediated by hepatic inflammation. Hepatic fat accumulation induces inflammation in hepatic cells which is the important mediator of fibrogenesis. ${ }^{8,20}$ It needs to note that the relationship between dietary patterns and hepatic fibrosis might be mediated by hepatic steatosis. Hence, hepatic steatosis status is considered as a potential confounder in studies of diet and hepatic fibrosis.

The components of prudent and Western dietary patterns in the "Health Professionals Follow-up Study" and healthy and Western dietary patterns in the "Swedish Mammography Cohort Study" showed similarities with the healthy and Western dietary patterns identified in our data. ${ }^{21,22}$ Therefore, healthy and Western (unhealthy) dietary patterns are likely to be similar among different populations which have shown to follow the same trends.

The healthy dietary pattern in our study included a high intake of low-fat dairies, white meat, nuts, vegetables, fruits, and vegetable oils. The adherence to this pattern was associated with lower risk of hepatic fibrosis. This relationship might be mediated by the healthy lifestyle factors related to this pattern. However, this association remained significant after adjustment for potential confounders, such as physical activity and smoking, and educational status. It needs to note that more tendencies toward healthier foods are observed subsequent to intermediary events related to NAFLD such as dyslipidemia, diabetes, and obesity. This phenomenon would tend to weaken the association between the healthy dietary pattern and fibrosis. Data on the association between dietary pattern and risk of hepatic fibrosis are sparse. In accordance with our findings, Katasagoni et al observed that adherence to a healthy dietary pattern (low-fat dairies, vegetables, and fish) combined with optimal sleep duration shows an inverse association with liver stiffness. ${ }^{23}$ Interestingly, in spite of the healthy dietary pattern, the food groups in this pattern individually showed no association with the risk of hepatic fibrosis. Therefore, the association between healthy dietary and hepatic fibrosis could be attributable to cumulative effects of foods in this pattern. The healthy dietary patterns focus mainly on plant-based foods and white meat. These foods contain a variety of nutraceuticals such as phytosterols, polyphenols, and omega-3 fatty acids which could downregulate hepatic inflammation through antioxidant and anti-inflammatory properties. ${ }^{24-26}$

The Western dietary pattern derived from our data included a high intake of refined grains, potato, red meat, white meat, eggs, and soft drinks. This pattern was associated with an increased risk of hepatic fibrosis. This association remained significant after adjustment for steatosis status, indicating that Western pattern acts directly on hepatic fibrogenesis. It is well established that refined grains, soft drinks, and red meat are associated with features of NAFLD. ${ }^{14,27}$ Among them, we observed that red meat and soft drinks were associated with an increased risk of hepatic fibrosis independent of steatosis. Red meat is a rich source of cholesterol and 
long-chain saturated fatty acids (palmitate and stearate), and a poor source of antioxidants and dietary fiber. On the other hand, soft drinks are considered to be one of the rich sources of fructose in Western diets and have an important role in the pathogenesis of NAFLD, independent of other risk factors. Katasagoni et al reported that the adherence to unhealthy dietary pattern with high consumption of high-fat dairies, refined cereals, potato, and red meats with television viewing had no association with liver stiffness. ${ }^{23}$ This inconsistency with our results may be due to the inclusion of soft drinks in the Western pattern derived in our data, while no soft drink intake was found in the study reported by Katasagoni. In this regard, Abdelmalek et al observed that large consumption of fructose had a positive association with the fibrosis severity among NAFLD patients. ${ }^{28}$ It is suggested that the Western dietary pattern, with its pro-inflammatory properties, could promote hepatic inflammation and fibrogenesis through the Kupffer cells and hepatic stellate cell activation. ${ }^{8,29}$

The Iranian dietary pattern in our study included a high intake of high-fat dairies, refined grains, legumes, white meat, sugars, coffee and tea, and hydrogenated fats. This pattern was similar to Iranian dietary patterns identified in other studies in the Iranian population. ${ }^{30-32}$ Unlike our expectation, the adherence to this pattern was not associated with an increased risk of hepatic fibrosis. This finding can be associated with the natural complexity of the Iranian dietary pattern which is loaded with both healthy and unhealthy foods. Present evidence indicates that hydrogenated fats, sugars, and refined grains have been linked with features of NAFLD. ${ }^{27,33}$ However, our data elucidated that only hydrogenated fats were associated with an increased risk of hepatic fibrosis independent of hepatic steatosis. Hydrogenated fatty acids are the main source of trans-fatty acids in the Iranian regimes. Trans-fatty acids in addition to insulin resistance could induce oxidative stress which is the main contributor to hepatic fibrosis via the activation of hepatic stellate cells and Kupffer cells. ${ }^{34,35}$ On the other hand, the Iranian dietary pattern emphasizes on tea and coffee consumption, which potentially contain a wide variety of antioxidant compounds. Our results reproduce other reports that coffee and tea consumption is associated with a lower risk of hepatic fibrosis. ${ }^{36,37}$ Therefore, consumption of tea and coffee could interfere with hydrogenated fats in this pattern and counteract with their effects on liver fibrosis. In accordance with our finding, Esmaillzadeh et al observed that the Iranian dietary pattern, which emphasizes on refined and whole grains, potato, hydrogenated fats, legumes, teas, and broth, had no association with NAFLD risk factors. ${ }^{31}$
The limitations involved in the present study were as follows: first, cross-sectional studies (such as the current research) are unable to infer the causal relationship between diet and diseases. Therefore, our findings need to be evaluated in the prospective cohort studies. Second, three 3-DRs might be insufficient for evaluation of usual intakes but could measure the actual intakes and eliminate recall error. Third, the factor analysis method is implemented with several arbitrary decisions such as food grouping, the number of retained factors, type of rotation, and the naming of factors. However, it is important to note that factor analysis has been proved as a suitable and acceptable method in the extraction of dietary patterns for studying the diet-disease association. ${ }^{10}$

\section{Conclusion}

The current study showed that the adherence to the healthy dietary pattern which emphasizes high intake of low-fat dairies, white meat, nuts, vegetables, fruits, and vegetable oils combined with coffee and tea consumption might be helpful in the nutritional strategies against hepatic fibrosis. In future studies, the addition of eating behaviors and sleeping habits in the analysis of dietary patterns is recommended.

\section{Acknowledgments}

We would like to appreciate the participants and assistants for their help in this study. All the financial support was provided by Mashhad University of Medical Sciences, Mashhad, Iran.

\section{Disclosure}

The authors report no conflicts of interest in this work.

\section{References}

1. Birkenfeld AL, Shulman GI. Nonalcoholic fatty liver disease, hepatic insulin resistance, and type 2 diabetes. Hepatology. 2014;59(2):713-723.

2. Anderson EL, Howe LD, Jones HE, Higgins JPT, Lawlor DA, Fraser A. The prevalence of non-alcoholic fatty liver disease in children and adolescents: a systematic review and meta-analysis. PLoS One. 2015;10(10): e0140908.

3. Younossi ZM, Koenig AB, Abdelatif D, Fazel Y, Henry L, Wymer M. Global epidemiology of nonalcoholic fatty liver disease-Meta-analytic assessment of prevalence, incidence, and outcomes. Hepatology. 2016;64(1):73-84.

4. Chalasani N, Younossi Z, Lavine JE, et al. The diagnosis and management of non-alcoholic fatty liver disease: Practice guideline by the American Association for the Study of Liver Diseases, American College of Gastroenterology, and the American Gastroenterological Association. Hepatology. 2012;55(6):2005-2023.

5. Singh S, Allen AM, Wang Z, Prokop LJ, Murad MH, Loomba R. Fibrosis progression in nonalcoholic fatty liver vs nonalcoholic steatohepatitis: a systematic review and meta-analysis of paired-biopsy studies. Clin Gastroenterol Hepatol. 2015;13(4):643-654.

6. Sarwar R, Pierce N, Koppe S. Obesity and nonalcoholic fatty liver disease: current perspectives. Diabetes Metab Syndr Obes. 2018;11:533-542. 
7. Seko Y, Nishikawa T, Umemura A, et al. Efficacy and safety of canagliflozin in type 2 diabetes mellitus patients with biopsy-proven nonalcoholic steatohepatitis classified as stage 1-3 fibrosis. Diabetes Metab Syndr Obes. 2018;11:835-843.

8. Yu J, Marsh S, Hu J, Feng W, Wu C. The pathogenesis of nonalcoholic fatty liver disease: interplay between diet, gut microbiota, and genetic background. Gastroenterol Res Pract. 2016;2016(6):1-13.

9. Jacques PF, Tucker KL. Are dietary patterns useful for understanding the role of diet in chronic disease? Am J Clin Nutr. 2001;73(1):1-2.

10. Hu FB. Dietary pattern analysis: a new direction in nutritional epidemiology. Curr Opin Lipidol. 2002;13(1):3-9.

11. Aller R, Izaola O, de La Fuente B, de Luis Román DA. Mediterranean diet is associated with liver histology in patients with non alcoholic fatty liver disease. Nutr Hosp. 2015;32(6):2518-2524.

12. Hekmatdoost A, Shamsipour A, Meibodi M, Gheibizadeh N, Eslamparast T, Poustchi H. Adherence to the Dietary Approaches to Stop Hypertension (DASH) and risk of nonalcoholic fatty liver disease. Int J Food Sci Nutr. 2016;67(8):1024-1029.

13. Ryan MC, Itsiopoulos C, Thodis $\mathrm{T}$, et al. The Mediterranean diet improves hepatic steatosis and insulin sensitivity in individuals with non-alcoholic fatty liver disease. J Hepatol. 2013;59(1):138-143.

14. Oddy WH, Herbison CE, Jacoby P, et al. The Western dietary pattern is prospectively associated with nonalcoholic fatty liver disease in adolescence. Am J Gastroenterol. 2013;108(5):778-785.

15. Williams B, Onsman A, Brown T. Exploratory factor analysis: a fivestep guide for novices. Aust J Paramed. 2010;8(3):1.1-1.13.

16. Fraquelli M, Rigamonti C, Casazza G, et al. Reproducibility of transient elastography in the evaluation of liver fibrosis in patients with chronic liver disease. Gut. 2007;56(7):968-973.

17. Abenavoli L, Beaugrand M. Transient elastography in non-alcoholic fatty liver disease. Ann Hepatol. 2012;11(2):172-178.

18. Sasso M, Beaugrand M, De Ledinghen V, et al. Controlled attenuation parameter (CAP): a novel VCTE ${ }^{\mathrm{TM}}$ guided ultrasonic attenuation measurement for the evaluation of hepatic steatosis: preliminary study and validation in a cohort of patients with chronic liver disease from various causes. Ultrasound Med Biol. 2010;36(11):1825-1835.

19. Selem SS, Castro MA, Cesar CL, Marchioni DM, Fisberg RM. Associations between dietary patterns and self-reported hypertension among Brazilian adults: a cross-sectional population-based study. J Acad Nutr Diet. 2014;114(8):1216-1222.

20. Seki E, Schwabe RF. Hepatic inflammation and fibrosis: functional links and key pathways. Hepatology. 2015;61(3):1066-1079.

21. Strate LL, Keeley BR, Cao Y, Wu K, Giovannucci EL, Chan AT. Western dietary pattern increases, and prudent dietary pattern decreases, risk of incident diverticulitis in a prospective cohort study. Gastroenterology. 2017;152(5):1023-1030.

22. Khani BR, Ye W, Terry P, Wolk A. Reproducibility and validity of major dietary patterns among Swedish women assessed with a food-frequency questionnaire. J Nutr. 2004;134(6):1541-1545.
23. Katsagoni CN, Papatheodoridis GV, Papageorgiou MV, et al. A "healthy diet-optimal sleep" lifestyle pattern is inversely associated with liver stiffness and insulin resistance in patients with nonalcoholic fatty liver disease. Appl Physiol Nutr Metab. 2017;42(3):250-256.

24. Scicchitano P, Cameli M, Maiello M, et al. Nutraceuticals and dyslipidaemia: beyond the common therapeutics. J Funct Foods. 2014;6:11-32.

25. Sears B. Anti-inflammatory diets. J Am Coll Nutr. 2015;34(Suppl 1): 14-21.

26. Salomone F, Godos J, Zelber-Sagi S. Natural antioxidants for nonalcoholic fatty liver disease: molecular targets and clinical perspectives. Liver Int. 2016;36(1):5-20.

27. Mirmiran P, Amirhamidi Z, Ejtahed HS, Bahadoran Z, Azizi F. Relationship between diet and non-alcoholic fatty liver disease: a review article. Iran J Public Health. 2017;46(8):1007-1017.

28. Abdelmalek MF, Suzuki A, Guy C, et al. Increased fructose consumption is associated with fibrosis severity in patients with nonalcoholic fatty liver disease. Hepatology. 2010;51(6):1961-1971.

29. Tomita K, Teratani T, Suzuki T, et al. Free cholesterol accumulation in hepatic stellate cells: mechanism of liver fibrosis aggravation in nonalcoholic steatohepatitis in mice. Hepatology. 2014;59(1):154-169.

30. Mirmiran P, Bahadoran Z, Vakili AZ, Azizi F. Western dietary pattern increases risk of cardiovascular disease in Iranian adults: a prospective population-based study. Appl Physiol Nutr Metab. 2017;42(3):326-332.

31. Esmaillzadeh A, Azadbakht L. Major dietary patterns in relation to general obesity and central adiposity among Iranian women. $J$ Nutr. 2008;138(2):358-363.

32. Azizi H, Asadollahi K, Davtalab Esmaeili E, Mirzapoor M. Iranian dietary patterns and risk of colorectal cancer. Health Promot Perspect. 2015;5(1):72-80.

33. Georgoulis M, Kontogianni MD, Tileli N, et al. The impact of cereal grain consumption on the development and severity of non-alcoholic fatty liver disease. Eur J Nutr. 2014;53(8):1727-1735.

34. Kohli R, Kirby M, Xanthakos SA, et al. High-fructose, medium chain trans fat diet induces liver fibrosis and elevates plasma coenzyme Q9 in a novel murine model of obesity and nonalcoholic steatohepatitis. Hepatology. 2010;52(3):934-944.

35. Dhibi M, Brahmi F, Mnari A, et al. The intake of high fat diet with different trans fatty acid levels differentially induces oxidative stress and non alcoholic fatty liver disease (NAFLD) in rats. Nutr Metab. 2011;8(1):65.

36. Anty R, Marjoux S, Iannelli A, et al. Regular coffee but not espresso drinking is protective against fibrosis in a cohort mainly composed of morbidly obese European women with NAFLD undergoing bariatric surgery. J Hepatol. 2012;57(5):1090-1096.

37. Marventano S, Salomone F, Godos J, et al. Coffee and tea consumption in relation with non-alcoholic fatty liver and metabolic syndrome: a systematic review and meta-analysis of observational studies. Clin Nutr. 2016;35(6):1269-1281. 


\section{Supplementary materials}

Table SI Food grouping used in the dietary pattern analyses

\begin{tabular}{|l|l|}
\hline Food groups & Food items \\
\hline Refined grains & White bread, rice, flour, macaroni, noodle, biscuit, cake \\
\hline Whole grains & Barely bread, whole wheat, wheat germ, oat, bulgur, corn flakes \\
\hline Red meat & Beef, lamb, camel, sausages, hamburger, processed meats, organ meats \\
\hline White meat & Chicken, turkey, ostrich, fish, seafood \\
\hline High-fat dairies & Cream, butter, ice cream, pizza cheese \\
\hline Low-fat dairies & Yogurt, dough (yogurt drink), milk, cheese, curd \\
\hline Legumes & Beans, chickpeas, lima beans, broad beans, lentil, soy \\
\hline Nuts & Peanut, almond, pistachio, walnut, hazelnut, roasted seeds \\
\hline Potato & Potato \\
\hline Eggs & Eggs \\
\hline Soft drinks & Carbonated drinks, artificial juice \\
\hline Vegetable oils & Vegetable oils \\
\hline Hydrogenated fats & Animal fats, hydrogenated vegetable oils \\
\hline Vegetables & $\begin{array}{l}\text { Cucumber, tomato, spinach, pepper, mushroom, garlic, carrot, onions, mixed vegetables, lettuce, cabbage, eggplant, celery, } \\
\text { green peas, green beans, turnip, corn, tomato paste }\end{array}$ \\
\hline Fruits & $\begin{array}{l}\text { Apple, cherries, grapefruit, apricots, plum, kiwi, strawberry, oranges, grapes, berries, dates, barberry, banana, pomegranate, } \\
\text { melon, naringin, lemon, raisin, mulberry, persimmon, peach, cantaloupe, watermelon, pear, fruit juices }\end{array}$ \\
\hline Sugars & Jam, honey, candy, sugar, chocolate, pastry \\
\hline Coffee and tea & Black tea, green tea, coffee \\
\hline
\end{tabular}

Table S2 Multivariable-adjusted $\mathrm{OR}^{\text {a }}$ for hepatic fibrosis across tertiles of food groups

\begin{tabular}{|c|c|c|c|c|}
\hline Food groups & Tertile I & Tertile 2 & Tertile 3 & $P$-trend \\
\hline Low-fat dairies & Ref & $0.45(0.14-1.5)$ & $0.40(0.13-1.2)$ & 0.26 \\
\hline High-fat dairies & Ref & $1.18(0.5 \mathrm{I}-1.49)$ & $\mathrm{I} .97(0.78-3.6 \mathrm{I})$ & 0.31 \\
\hline Red meat & Ref & $1.21(0.70-2.25)$ & $2.19(1.21-3.74)$ & 0.04 \\
\hline White meat & Ref & $1.48(0.52-4.19)$ & $1.26(0.42-3.8)$ & 0.75 \\
\hline Refined grains & Ref & $\mathrm{I} .27(0.6 \mathrm{I}-2.73)$ & $2.08(0.86-4.94)$ & 0.27 \\
\hline Potato & Ref & $0.88(0.54-1.57)$ & $\mathrm{I} .34(0.88-2.3 \mathrm{I})$ & 0.65 \\
\hline Eggs & Ref & $1.14(0.56-2.44)$ & $1.42(0.68-2.70)$ & 0.47 \\
\hline Legumes & Ref & $0.68(0.38-1.48)$ & $0.99(0.52-1.87)$ & 0.31 \\
\hline Nuts & Ref & $0.89(0.67-|.3|)$ & $0.73(0.5 \mathrm{I}-\mathrm{I} .07)$ & 0.06 \\
\hline Vegetables & Ref & $0.91(0.3-2.7)$ & $0.49(0.15-1.5)$ & 0.41 \\
\hline Fruits & Ref & $0.57(0.16-1.8)$ & $0.47(0.14-1.5)$ & 0.43 \\
\hline Coffee and tea & Ref & $0.69(0.4 I-I .18)$ & $0.38(0.17-0.7 I)$ & 0.03 \\
\hline Sugars & Ref & $\mathrm{I} .24(0.95-I .7 \mathrm{I})$ & I.4I (0.96-I.85) & 0.07 \\
\hline Soft drinks & Ref & $1.18(0.58-2.34)$ & $2.21(1.15-4.23)$ & 0.02 \\
\hline Vegetable oil & Ref & $1.02(0.45-1.92)$ & $0.68(0.26-1.38)$ & 0.71 \\
\hline Hydrogenated oil & Ref & $1.87(1.16-3.37)$ & $2.58(1.48-4.98)$ & 0.01 \\
\hline
\end{tabular}

Notes: aMultivariable-adjusted ORs $(95 \% \mathrm{Cl})$ were obtained using binary logistic regression. Adjusted for sex, age, BMI, WC, smoking, education, diabetes, physical activity, hepatic steatosis status, energy intake, and anti-diabetic drugs and dietary supplement use. Abbreviations: BMI, body mass index; WC, waist circumference.

Diabetes, Metabolic Syndrome and Obesity: Targets and Therapy is an international, peer-reviewed open-access journal committed to the rapid publication of the latest laboratory and clinical findings in the fields of diabetes, metabolic syndrome and obesity research. Original research, review, case reports, hypothesis formation, expert Submit your manuscript here: https://www.dovepress.com/diabetes-metabolic-syndrome-and-obesity-targets-and-therapy-journal opinion and commentaries are all considered for publication. The manuscript management system is completely online and includes a very quick and fair peer-review system, which is all easy to use. Visit $\mathrm{http}: / /$ www.dovepress.com/testimonials.php to read real quotes from published authors. 